\title{
Geometric Limits on Spatial Resolution for Cs-Corrected STEM Analysis of Thick TEM Lamellae
}

J. Bruley, $*$ J. Ringnalda**

* IBM T.J. Watson Research Center, PO Box 218, Yorktown Heights, NY 10598

** FEI Company, Hillsboro, Oregon 97124, and The Ohio State University, Columbus, Ohio 43210

This study addresses analytical spatial resolution of samples in the 50-100nm thickness range when examined using a high convergence (semi-angle $\sim 30 \mathrm{mrad}$ ) beam such as would be associated with a $\mathrm{C}_{\mathrm{s}}$ corrected STEM. Achieving the highest spatial resolution microanalysis requires extremely thin samples, typically foils less than $200 \AA$ are called for. There have been numerous studies describing the elastic and inelastic scattering mechanisms that broaden the probe profile as it traverses the TEM sample. In the microelectronics industry, many samples are prepared using the FIB and are often somewhat thicker than $500 \AA$, especially if they are from isolated and electrically tested devices. Despite being too thick there is still significant interest to characterize such samples and an estimate of the expected resolution is called for.

Two samples of Si CMOS gate stacks were inspected. One gate stack comprised of a TiN metal gate electrode with $\mathrm{Al}_{2} \mathrm{O}_{3}$ on a thin $\mathrm{SiO}_{2}$ gate oxide layer on $\mathrm{Si}$, the second had the same metal electrode and an $\mathrm{HfO}_{2} / \mathrm{SiO}_{2}$ gate oxide. In this case samples were prepared by polish edge to enable a range of sample thicknesses to be explored. Thickness was estimated using valence loss EELS. The microscope was a $\mathrm{C}_{\mathrm{s}}$ corrected STEM Titan 80-300 microscope installed at McMaster University. Angle of convergence was changed by changing the angle limiting final condenser lens aperture. The stage height was adjusted to bring the Si lattice into focus and thereafter the objective current was used to bring the high-K dielectric into "best" focus as could be assessed on a reduced area raster.

The principal finding of this study is that despite being able to bring into focus and resolve the Si [110] dumbbells in the HAADF STEM images enhanced by channeling, even in this high thickness range there is a different optimum focus for achieving the sharpest images and highest resolution microanalysis of the amorphous high atomic number gate oxide material. This cautions against assuming the best spatial resolution for analysis is achieved when the $\mathrm{Si}$ is in sharp focus for samples of about $50 \mathrm{~nm}$. It also indicates that the volume analyzed is not probe size limited to sub $2 \AA$.

A large contribution to this effective probe size change is believed to be associated with the wide beam convergence. For small convergence angles $(<10 \mathrm{mrad})$ the focus and thickness effects shown here are not large enough to be readily detected but at angles of $>20 \mathrm{mrad}$ and thickness $>50 \mathrm{~nm}$ the effective beam diameter can change by as much as $5 \AA$ due to geometry alone. This is significant for analytical systems that allow large convergence angles to be used.

\section{References}

[1] G. Botton, for discussions and making available the TITAN at the Canadian Centre for Electron Microscopy, McMaster University, Canada 

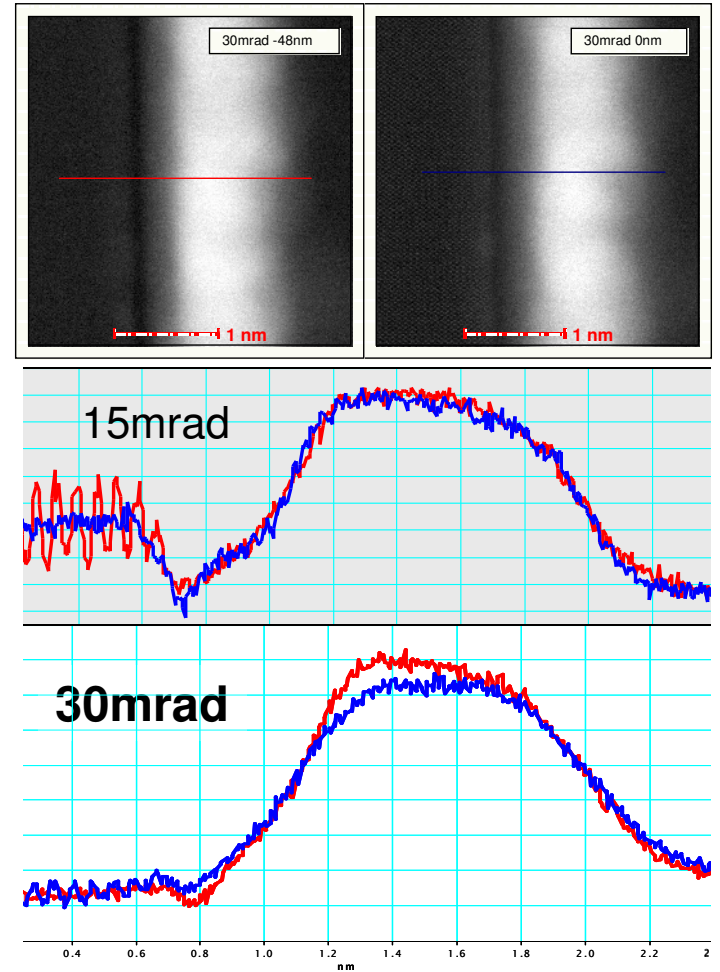

Fig. 1: HAADF images of 80nm sample "focused" on Si (0nm defocus) and high-K (45nm defocus). The $30 \mathrm{mrad}$ beam $\& 45 \mathrm{~nm}$ defocus results in $\sim 2 \AA$ broadening. For $15 \mathrm{mrad}$, defocus effect is barely discernable on the high-K but leads to loss of Si resolution
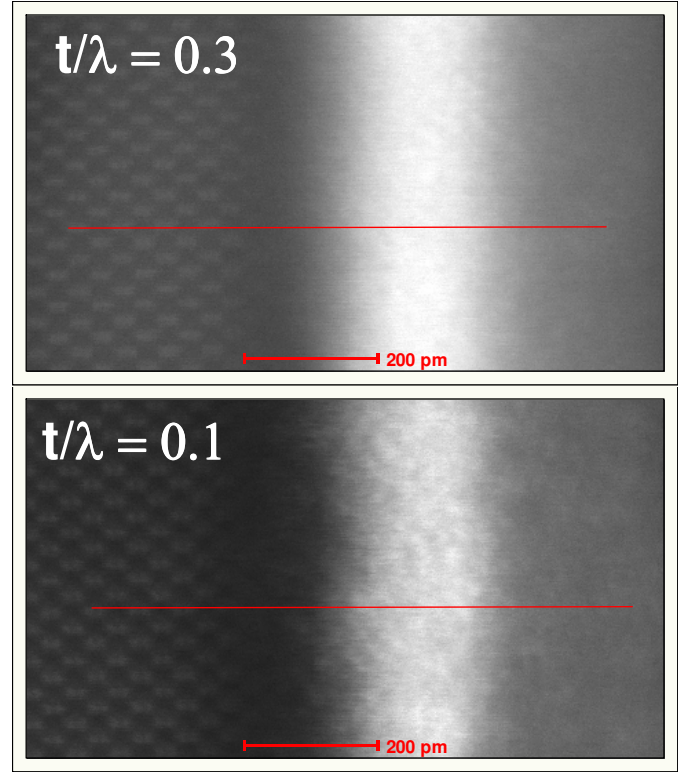

Fig 2: Sample thickness effect with large convergence angle is readily discernable on high $\mathrm{K}$ material. Optimum focus for high $\mathrm{K}$ is $\sim 0.5 \mathrm{x}$ sample thickness for $>20 \mathrm{mrad}$ and $>50 \mathrm{~nm}$ foils
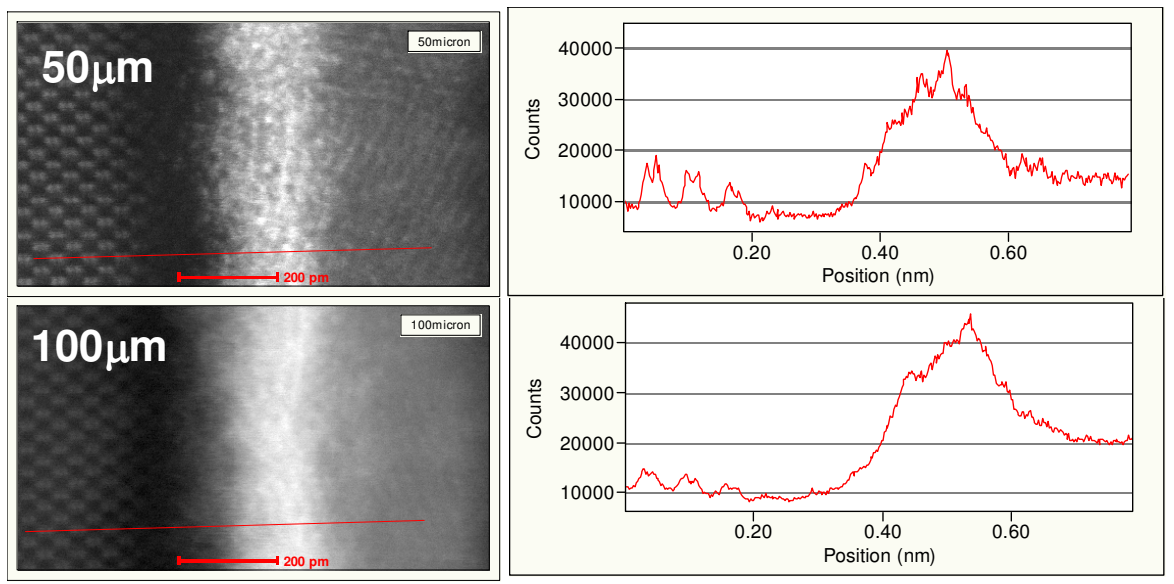

Fig. 3. Selection of smaller aperture leads to improved resolution in high-K material. Larger aperture still enables Si dumbbell resolution but degraded resolution on high-K 